\title{
THE BRAZIER EFFECT AND ITS INFLUENCE IN THE DESIGN OF BEAMPIPES FOR PARTICLE COLLIDERS
}

\author{
J. Knaster ${ }^{1}$, G. Tortora ${ }^{2}$, R. Veness ${ }^{1}$
}

The Brazier effect describes the influence of ovalisation on the buckling of thin shells. Ovalisation can be enhanced by applied bending moments and internal or external pressure. This can limit the design of vacuum chambers, requiring the addition of supports or changes in wall thickness. Since this is a non-linear effect, finite elements packages can fail to give good results. A theoretical and empirical approach is performed showing the results and conclusions of the tests.

1 Accelerator Technology Division, CERN, Geneva, Switzerland

2 Department of Engineering, Oxford University, UK

Published in Vacuum 64, Issue 2 (2001)

Administrative Secretariat AT Division CERN

CH - 1211 Geneva 23
Geneva, Switzerland

29 July 2003 
The Brazier effect and its influence in the design of beampipes for particle colliders

\author{
J. Knaster CERN, Geneva, Switzerland \\ G. Tortora Department of Engineering, Oxford University \\ R. Veness CERN, Geneva, Switzerland
}

\begin{abstract}
The Brazier effect describes the influence of ovalisation on the buckling of thin shells. Ovalisation can be enhanced by applied bending moments and internal or external pressure. This can limit the design of vacuum chambers, requiring the addition of supports or changes in wall thickness. Since this is a non-linear effect, finite elements packages can fail to give good results. A theoretical and empirical approach is performed showing the results and conclusions of the tests.
\end{abstract}

\title{
1. Introduction
}

Vacuum chambers for interaction regions of particle colliders pass physically through the centre of the particle physics experiments [1]. They must be highly optimised mechanical structures to minimised their impact on physics performance.

These experimental beam pipes can be assumed to be a long, thin shell, with vacuum inside and atmospheric pressure on the outside. In addition to this pressure load, allowances must also be made for some bending moment load, partly due to the self-weight of the tube and partly to bending moments induced from supports and misalignment. The use of finite elements methods is not straightforward since there are some non-linear effects due to the ovalisation of the beam under bending.

The aim of this study is to investigate the structural behaviour of long, thin, elastic tubes subjected to bending moments and external pressure, taking into consideration the effect of the ovalization of the tube section due to the bending moment. This is the so-called Brazier effect [2].

\section{The Brazier effect in the absence of a pressure load}

\subsection{Preliminary discussion on the effects of a varying cross-section}

As an initially straight tube is bent the tensile and compressive loads tend to "flatten" or ovalise the section. It will be assumed that the tube is initially perfectly circular and that, under bending, its cross-section deforms into a perfect ellipse. From elementary beam theory it is known that:

$$
\mathrm{M}=\mathrm{CEI}
$$

where $\mathrm{M}$ is the bending moment, $\mathrm{C}$ is the curvature of the beam and $\mathrm{El}$ the flexural stiffness with $E$ being the Young modulus and I the 2nd moment of inertia. A first approximation would assume that $\mathrm{El}$ is a constant and hence the relationship between $\mathrm{M}$ and $\mathrm{C}$ is linear. On the other hand, if notice is taken of the variation in cross-section, I is not a constant any longer. In particular, as the curvature increases, the ovalisation of the tube also increases leading to larger reductions in I. Hence it seems reasonable to expect the section to reach a maximum $M$ at some critical value of curvature. This is the effect Brazier studied in his 1927 paper, and the resulting maximum moment will be referred to as the Brazier moment $M_{b r a z}$.

It seems sensible to compare the Brazier moment to the classical buckling moment $M_{\text {buck }}$ for the section assuming no ovalisation. The latter given by the equation: 
$\mathrm{M}_{\text {buck }}=\frac{\pi}{\sqrt{3}}$ Eath

where $a$ is the radius, $t$ is the thickness and $h$ is the effective thickness of the tube which equals

$\mathrm{h}=\frac{\mathrm{t}}{\sqrt{1-v^{2}}}$

2.2 The Brazier moment: a quantitative analysis

\subsubsection{Aims and strategy}

First we will relate the section's ovalisation to the reduction in I. Then the ovalisation , will be related to curvature and moment. The strategy is to find an expression for the strain energy of the tube $U$ in terms of , and $\mathrm{C}$ and these can then be related to each other since $\mathrm{dU} / \mathrm{d}=0$ by the minimum strain energy principle. This is equivalent to saying that for a certain curvature the ovalisation is such as to minimise the internal energy state of the tube. Then an expression for $\mathrm{M}$ in terms of or $\mathrm{C}$ can be derived since $M=d U / d C$. Finally the maximum bending moment $M_{b r a z}$ can be found (and also the curvature and ovalisation at which it occurs). Much of this derivation follows from the work of Calledine [3] where further explanations can be found.

\subsubsection{The 2 nd moment of area of an oval section}

Let's assume that the section is initially perfectly circular and that, under bending, it shall deform into a perfect ellipse. Hence the expression for the section's radial displacement $w$ [4] is:

$$
\mathrm{w}=\mathrm{a} \zeta \cos 2 \theta
$$

Moreover it is assumed that the cross-section deforms inextensionally in its own plane. Hence if $v$ is the circumferential displacement:

$\frac{d \mathrm{v}}{d \theta}+\mathrm{w}=0$

Then

$\mathrm{v}=-\frac{1}{2} \mathrm{a} \zeta \sin 2 \theta$

Where the constant of integration has been set to zero since we are assuming no net rotation of the section about the axis of the tube.

Taking the former into consideration it can be shown that

$\mathrm{I}=\pi \mathrm{a}^{3} \mathrm{t}\left(1-\frac{3}{2} \zeta+\frac{5}{8} \zeta^{2}\right)$

which relates the 2 nd moment of area to the ovalisation

\subsubsection{The internal strain energy of a bent and ovalised section}

There are two separate contributions to the internal energy $\mathrm{U}$ : one is from the longitudinal bending (stretching) of the beam, the other is from the ovalisation of the section. In reality both these deformations occur at the same time. By means of the superposition principle, $U$ can be found by assuming that the initially circular tube "first ovalises without bending and then bends without ovalising". This yields the correct result because the final energy state is conservative depending only on the initial and final conditions. It is shown in [3] that the strain energy stored in a straight, ovalised section,$\cup_{1}$, is given by

$$
\mathrm{U}_{1}=\frac{3}{8 \mathrm{a}} \pi \mathrm{Et}^{3} \zeta^{2}
$$


and the internal energy due to bending without ovalisation, $\mathrm{U}_{2}$

$\mathrm{U}_{2}=\frac{1}{2} \mathrm{C}^{2} \mathrm{EI}$

So, adding both expressions

$\mathrm{U}=\frac{1}{2} \mathrm{C}^{2} \mathrm{E} \pi \mathrm{a}^{3} \mathrm{t}\left(1-\frac{3}{2} \zeta+\frac{5}{8} \zeta^{2}\right)+\frac{3}{8 \mathrm{a}} \pi \mathrm{Et}^{3} \zeta^{2}$

In his analysis Brazier disregarded the term ${ }^{2}$ in the bracket (i.e. the one coming from the expansion for I) and this approximation seems to give results in good agreement with experimental results, whilst simplifying the analysis greatly.

\subsubsection{The Brazier moment}

Using the minimum strain energy principle

$\frac{\partial \mathrm{U}}{\partial \zeta}=0$

we obtain

$\zeta=\frac{4 c^{2}}{3}$

where

$\mathrm{c}=\frac{\sqrt{3}}{2 \mathrm{~h}} \mathrm{Ca}^{2}$

c gives a dimensionless measure of curvature. It is also convenient to define a dimensionless moment $\mathrm{m}$

$\mathrm{m}=\frac{\mathrm{M}}{\mathrm{M}_{\text {buck }}}$

Finally from a previous equation a dimensionless energy coefficient can also be defined:

$\mathrm{u}=\mathrm{c}^{2}\left(1-\frac{3}{2} \zeta\right)+\frac{9}{16} \zeta^{2}$

And

$\mathrm{m}=2 \mathrm{c}\left(1-2 \mathrm{c}^{2}\right)$

or equally (see Figure 1)

$\mathrm{m}=\sqrt{3 \zeta}\left(1-\frac{3}{2} \zeta\right)$

The maximum of this function can then be found. It turns out that: 
$\mathrm{m}_{\text {max }}=\mathrm{m}_{\text {braz }}=0.544$

occurring at

$\mathrm{c}=0.408 \quad$ and $\quad \zeta=0.22$

The maximum value of the moment is the so called Brazier Moment $m_{\text {braz }}$.

Two observations can be made from this. Firstly, the Brazier effect seems to be very significant since the predicted failure moment is about half that which would be expected disregarding the ovalisation. Secondly, the maximum moment is expected to be reached after a large amount of ovalisation has taken place (around 20\%).

\subsubsection{Local buckling}

Failure can occur by local buckling at a moment smaller than Brazier's. To analyse the effective local buckling it is convenient to start from a criterion for stress at buckling and then compare this with an equation relating curvature and stress. The classical buckling stress is given by

$\sigma_{\text {buck }}=-\frac{E h}{\sqrt{3} \mathrm{a}}$

which is obtained by assuming that the cross section remains circular and setting the compressive stress equal to the stress level corresponding to classical buckling of a pipe under axial compression. Again it is convenient to define a dimensionless stress, as the ratio of the actual stress to the "classical" (i.e. no ovalisation assumed) buckling stress:

$\mathrm{s}=\frac{\sigma}{\sigma_{\text {buck }}}$

the minimum radius of curvature

$\rho=\frac{a}{1-3 \zeta}$

hence

$\mathrm{s}_{\mathrm{cr}}=1-3 \zeta$

and

$\mathrm{s}=2 \mathrm{c}(1-\zeta)$

2.3 Comparing the Brazier moment and the moment at failure by local buckling for the no pressure case

The condition for local buckling is that

$\mathrm{s}=\mathrm{s}_{\mathrm{cr}}$

And yields

$\mathrm{c}=0.33$

$\zeta=0.145$

$\mathrm{m}_{\text {buck }}=0.517$

A comparison with the values given for the Brazier moment in Section 2.2.4 shows that local buckling occurs at a moment slightly smaller than the Brazier moment, and that failure is expected to occur for 
smaller flattening $=0.145$. Note also that the buckling moment calculated taking into account ovalisation is about half of that calculated assuming no ovalisation.

\section{The effect of a pressure load}

The presence of a pressure load alters the calculations for both the Brazier moment and the moment for local buckling.

It has been given the expression for $u$ in the case of no pressure. It is evident that the term in $c^{2}$ is due to the bending of the tube, whereas the term in ${ }^{2}$ is due to the ovalisation of the section. The effect of superimposing a pressure is simply to increase the "ovalisation stiffness" by a factor $1+p / p^{*}$ where

$$
\mathrm{p} *=\frac{E \operatorname{th}^{2}}{4 \mathrm{a}^{3}}
$$

the pressure that would give buckling by mode 2 for a very long thin tube with free ends in the absence of a bending moment.

What has changed is the relationship between $\mathrm{c}$ and , so that:

$$
m=2 c\left(1-\frac{2 c^{2}}{1+\frac{p}{p^{*}}}\right)
$$

\subsection{The Brazier moment}

However in the case of the Brazier moment we are interested in the maximum moment a section can sustain at a given pressure obtaining the relationship

$$
1+\frac{p}{p^{*}}=6 c^{2}
$$

and substituting into the previous equation

$$
\mathrm{m}=0.544 \sqrt{1+\frac{\mathrm{p}}{\mathrm{p}^{*}}}
$$

This is the curve for the interaction diagram for the Brazier moment. Note that it gives the correct result for $p=0$

\subsection{The Local Buckling}

The relationship between $c$ and $1+p / p^{*}$ at failure is

$$
1-\frac{4 c^{2}}{1+\frac{p}{p^{*}}}=2 c\left(1-\frac{4 c^{2}}{3\left(1+\frac{p}{p^{*}}\right)}\right)
$$

Which yields 
$1+\frac{p}{p^{*}}=\frac{4 c^{2}(3-2 c)}{3(1-2 c)}$

which has to be compared with the similar expression obtained in section 3.1.

\subsection{The interaction diagram}

The interaction diagram has been plotted in Figure 2. It can be seen that the local buckling failure is more critical than the Brazier failure for $p / p^{*}>-0.6$. However, for all negative values of pressure the two failure moments are very similar.

\section{Experiments}

\subsection{Experimental Equipment}

\subsection{The tubes}

Tests were performed on commercially pure titanium tubes (Ti-40). The nominal outer diameter of the tubes was $63.50 .5 \mathrm{~mm}$, its thickness $0.540 .01 \mathrm{~mm}$ and the ovality tolerance $2 \%$. All of these parameters were carefully controlled for each tube.

A tube of free length $2 \mathrm{~m}$ was chosen, this compares with the minimum length for reasonable assumption of an infinitely long tube of $1.5 \mathrm{~m}$ [5].

\subsubsection{The apparatus}

The main issue with the apparatus was to devise a simple system of applying a pure moment to the tube. The tube was clamped to two vertical supports free to roll on bearings. Two threaded bars via a dynamometer connected the bases of the two supports. A load was applied by tightening the bars. The bearing made sure that only a pure moment was applied on the tube (minimum friction forces). The ends of the tube were sealed with ' $O$ '-rings and fitted with a pumping port. The apparatus is shown in the Figure 3

\subsection{Bending Without External Pressure}

\subsubsection{Method}

The aim of this test was to confirm the equations developed in Section 2, i.e. the expected ovalisation behaviour of an infinitely long, thin, elastic shell subjected to a pure bending moment load, with no pressure load

The tube was mounted on the supports, aligning the welding with the horizontal axis. This was done so that the ovalisation due to the bending would tend to increase the initial ovalisation rather than cancelling it out. The tube's outside diameter was measured at five points along the section. At each of these points the diameter was measured along the minor and major axes. The tube initial diameter was assumed to be equal to the average of the minor and major diameters in the absence of any loading. The displacement was then calculated taking as initial radius the actual initial radius at the

$\mathrm{w}=\mathrm{a} \zeta \cos 2 \theta$

section. Hence $\zeta$ could be found from

Note that this gives a different value for $\mathrm{w}$ along the minor and major axes, and so two different values of at the same section.

The tube was loaded gradually. Measurements were taken at 8 values of load. Each time a measurement of the deflection at the centre of the tube and a measurement of the plastic deflection at each loading was taken. Hence the tube was totally unloaded each time before going up to the next load. 


\subsubsection{Results}

Figure 4 shows plots of ovalisation versus applied moment for one of the four tested tubes. For each value of applied moment ten values of ovalisation are given. The measured values are also compared to the the theoretical values of the bending moment vs ovalisation given by dimensioning $\mathrm{m}$ As expected by the theory the Brazier effect could be tested only over a short range of moment: the Brazier moment for this tube is $\sim 950 \mathrm{Nm}$ ( $20 \%$ ovalisation) but plastic deformation starts at $\sim 220 \mathrm{Nm}$ ( $1 \%$ ovalisation). Hence, in the absence of a pressure load and for a tube of this geometry and this material, plastic yield is by far the most critical yielding criterium.

\subsection{Bending and pressure load test}

\subsubsection{Method}

The same apparatus was used to check the behaviour of the tubes when an external pressure load as well as a bending moment was applied. An extensive survey of the initial ovalisation at 21 different sections along each of the 6 tubes was carried out before the testing. The moment was applied, then the tube was placed inside a pneumatic overpressure test chamber with calibrated pressure gauge. The pressure in the chamber was gradually raised whilst the pressure inside the tube remained fixed to atmospheric. A manometer was connected to the inside of the tube via the pumping port to check for leaks.

Destructive tests were made on 6 tubes at two values of applied moment. The pressure in the test chamber was gradually increased in 0.05 bar steps until the tubes collapsed. The pressure was maintained for 60 seconds at each step.

\subsubsection{Preliminary theoretical analysis}

The buckling pressure was calculated using an accurate equation from [6] which applies for a tube with closed ends held circular under uniform external lateral and longitudinal pressure:

$$
\mathrm{p}_{\text {buck }}=\frac{\mathrm{E} \frac{\mathrm{t}}{\mathrm{a}}}{1+\frac{1}{2}\left(\frac{\pi \mathrm{a}}{\mathrm{n} 1}\right)^{2}}\left\{\frac{1}{\mathrm{n}^{2}\left[1+\frac{1}{2}\left(\frac{\pi \mathrm{a}}{\mathrm{n}}\right)^{2}\right]^{2}}+\frac{\mathrm{n}^{2} \mathrm{t}^{2}}{12 \mathrm{a}^{2}\left(1-v^{2}\right)}\left[1+\frac{1}{2}\left(\frac{\pi \mathrm{a}}{\mathrm{n} 1}\right)^{2}\right]^{2}\right\}
$$

the buckling mode is $n=2$. This equation predicts a buckling pressure of 1.51 bar.

\subsubsection{Results}

\begin{tabular}{|l|l|l|l|}
\hline TEST & $\begin{array}{l}\text { Applied Moment } \\
(\mathrm{Nm})\end{array}$ & $\begin{array}{l}\text { Collapse Pressure } \\
(\mathrm{bar})\end{array}$ & Comments \\
\hline 1 & 0 & 1.55 & \\
\hline 2 & 0 & 1.55 & $\begin{array}{l}\text { Failed between } 1.5 \text { and } \\
1.55 \text { ba }\end{array}$ \\
\hline 3 & 0 & 1.5 & Failed after 5" hold \\
\hline 4 & 0 & 1.35 & $\begin{array}{l}\text { Failed after 1.3 and } \\
1.35 \text { bar }\end{array}$ \\
\hline 5 & 265 & 1.3 & Failed after 50" hold \\
\hline 6 & 265 & 1.35 & Failed after 45" hold \\
\hline
\end{tabular}

Table 1. Results of pressure load tests

Figure 5 gives the results of the pressure tests. All the beams collapsed in buckling mode 2. The collapse was not perfectly symmetrical about the centre but seemed on first investigation to occur 
closer to the areas of higher initial ovalisation. The given moment of $265 \mathrm{Nm}$ is corrected to take into account the moment caused by the pressure on the ends of the tube, since the central deflection is not negligible. As the interaction diagram is only valid for elastic materials, higher moments were not applied.

\section{Summary and conclusions}

In the first part of this study equations have been developed to express the relation between induced ovalisation and applied bending moment in a thin, long, perfectly cylindrical tube. This ovalisationbuckling relation is the so-called Brazier effect. These equations have then been used to investigate the theoretical structural behaviour of such a shell when a pure bending moment is applied and when this bending moment is coupled with an external pressure.

Experimental tests have been carried out to verify the validity of these equations. In the first test only a pure bending was applied to the tube. In the tubes tested in this way plastic deformation occurred before much ovalisation could take place, and certainly long before the Brazier moment was reached: a moment causing a centre point deflection of $80 \%$ of the diameter would cause an ovalisation of less than $2 \%$.

In the second test an external pressure was coupled to the bending load. Tests were destructive and all tubes collapsed by buckling in mode 2. Experimental results were very close to the theoretical in the case of pure pressure loading. A small discrepancy between expected and actual results in the combined loading case was observed. This was probably due to the beam pipe undergoing some plastic deformation.

The applied bending moment of $265 \mathrm{Nm}$ is rather high, corresponding to the tube being supported with a $21 \mathrm{~m}$ span, and beyond the elastic moment threshold of $220 \mathrm{Nm}$. This moment was necessary to measure a significant decrease in buckling pressure. This of course indicates that, provided a beam pipe operates in its elastic region, the reduction in buckling pressure due to the application of bending moments is minimal, ie that the coupling between these two types of loading through ovalisation of the beam section is not significant. Moreover the analysis of section 4.2.2 seems to suggest that these results are fairly general.

Considering that by definition a beam pipe cannot operate with bending deflections higher than its radius, and due to the external pressure loads (according to formulas of buckling under external pressure, with appropriate safety factors for Beryllium R/t must be smaller than 60) the Brazier effect should not be an issue in the design of beam pipes for particle colliders.

\section{Acknowledgements}

This report would have not been possible without the help of Patrick Lepeule, Hendrik Kos, Tadeusz Kurtyka, Claude Hauviller, David Jenson, Gerhard Schneider, Blazej Skoczen, Claude Margaroli and Didier Chauville.

References

1. S. Karppinen, J. Knaster, P. Lepeule, G. Schneider and R. Veness, Design of Beampipes for LHC Experiments, IV Iberian Vacuum Meeting, Ávila July 2000

2. L.G. Brazier, Proc. Roy. Soc., London Series A, Vol. 116, p. 104, 1927

3. C.R Calledine, Theory of Shell Structures, Cambridge University Press

4. Timoshenko and Gere, Theory of Elastic Stability, McGraw Hill international editions, 2nd edition, 1963.

5. B.F Tatting, Z.Gurdal and V.V Vasiliev, The Brazier Effect for Finite Length Composite Cylinders under Bending, Int. J. Solids Structures Vol. 34 No.12, 1997, Elsevier Science Ltd..

6. W. Young, Roark's Formulas for Stress and Strain. Mc.Graw-Hill, 6th Edition, 1989. 
Figure 1. Relationship between dimensionless moment and ovalization in the absence of a pressure load

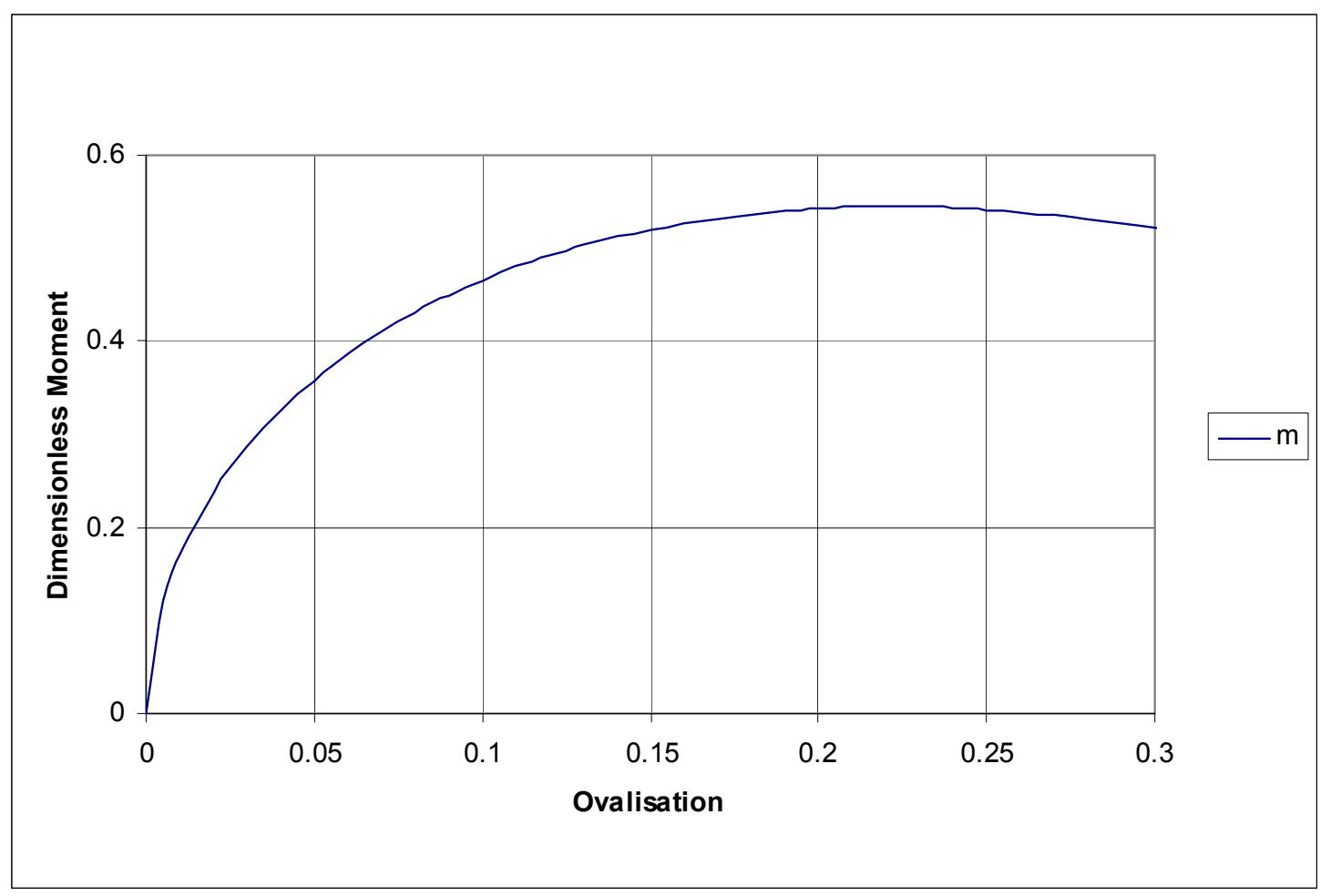


Figure 2. Variation of dimensionless moment at failure vs. dimensionless pressure.

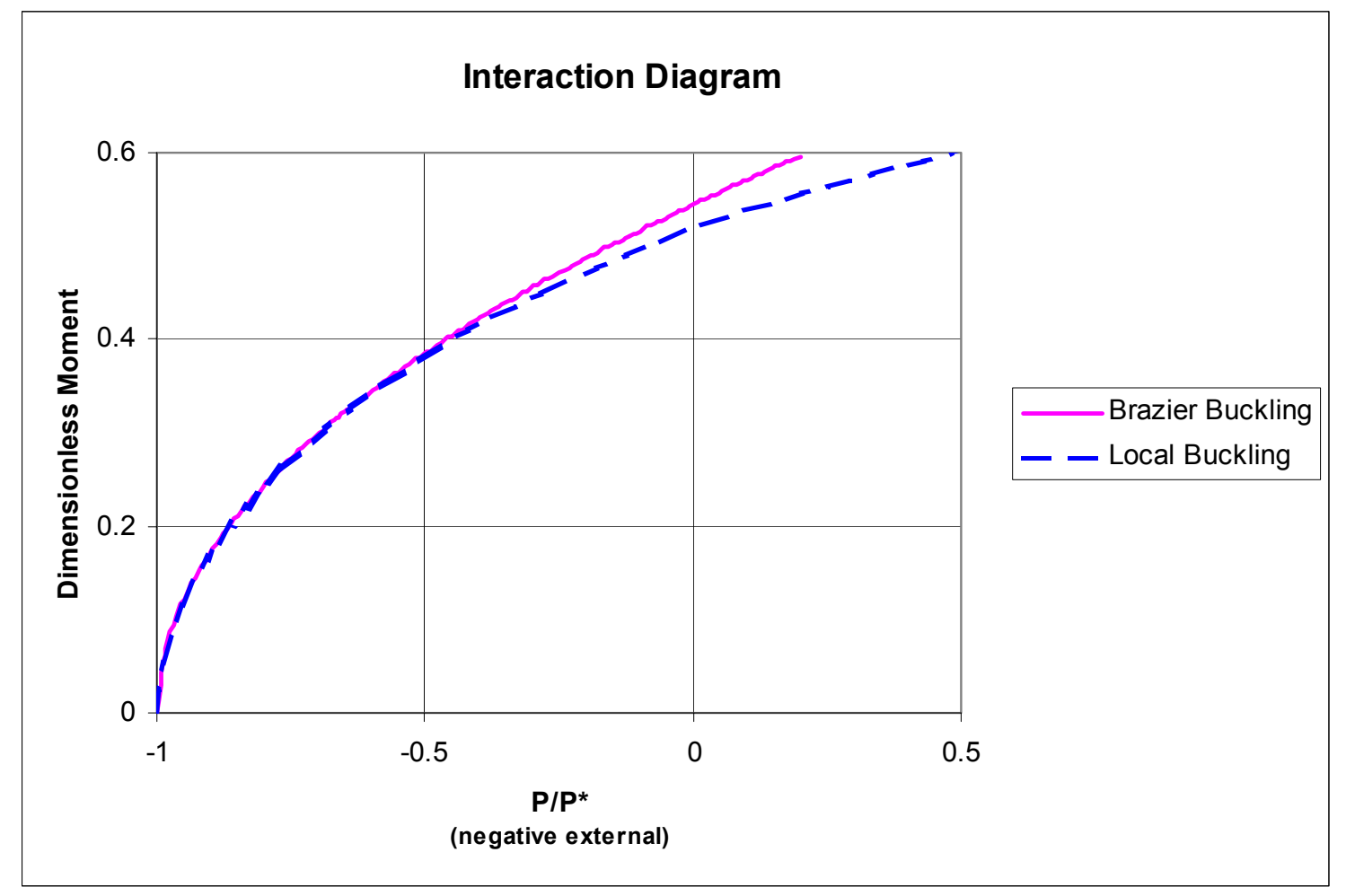


Figure 3. Sketch of the Experimental Apparatus

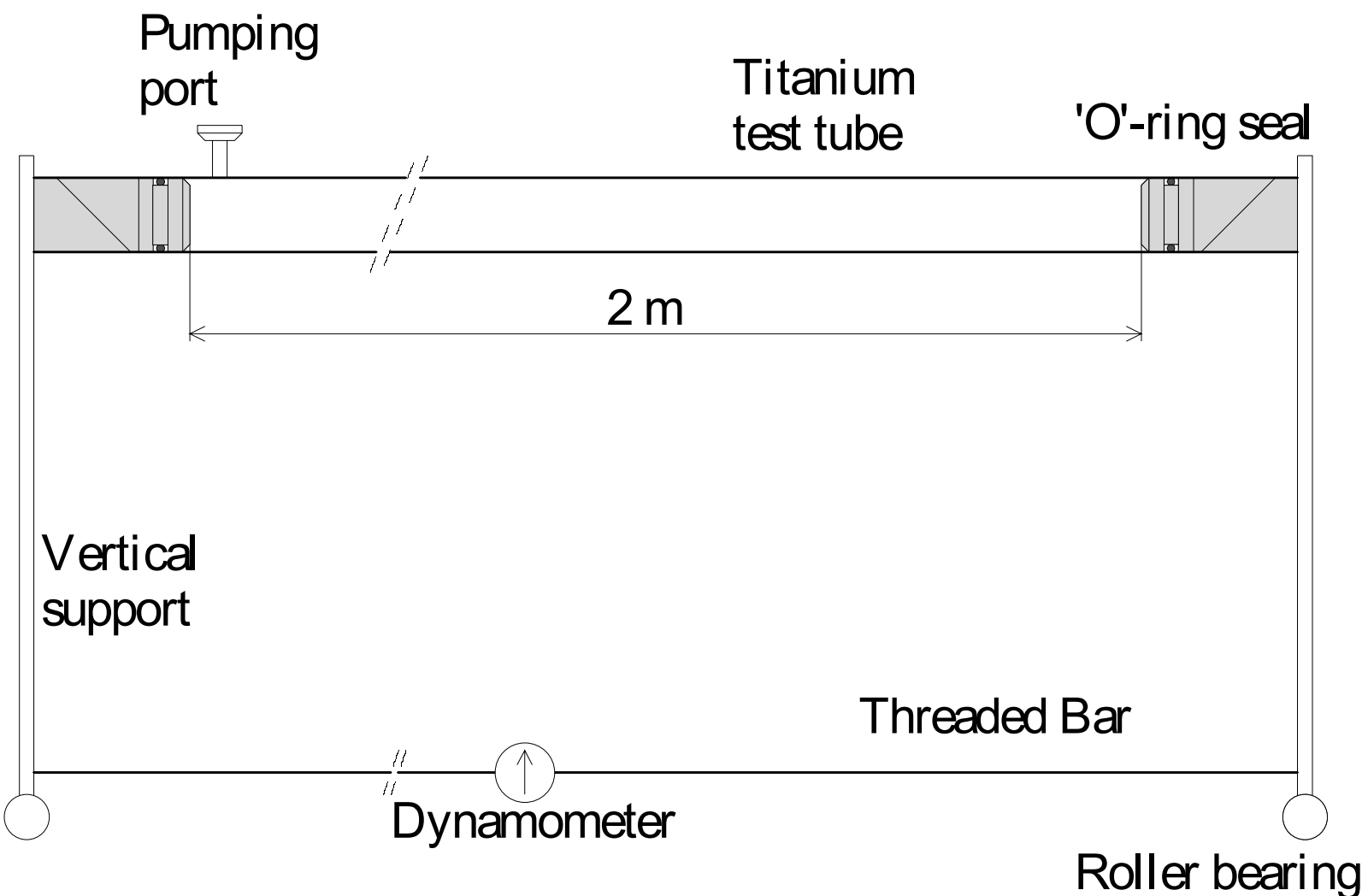


Figure 4. Applied bending moment vs measured ovalization. The continuous line shows the theoretical prediction

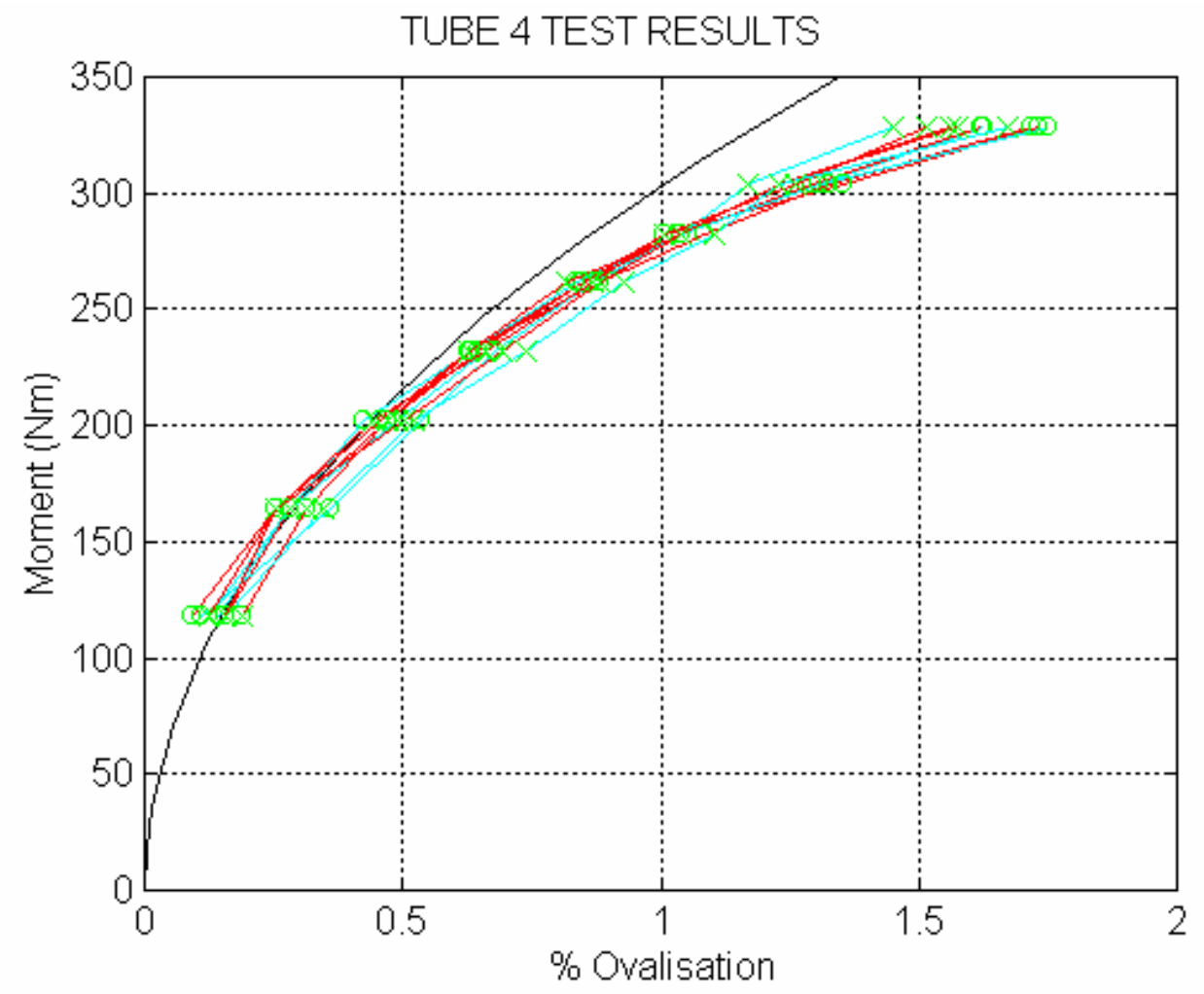


Figure 5. Theoretical and experimental buckling moment vs pressure

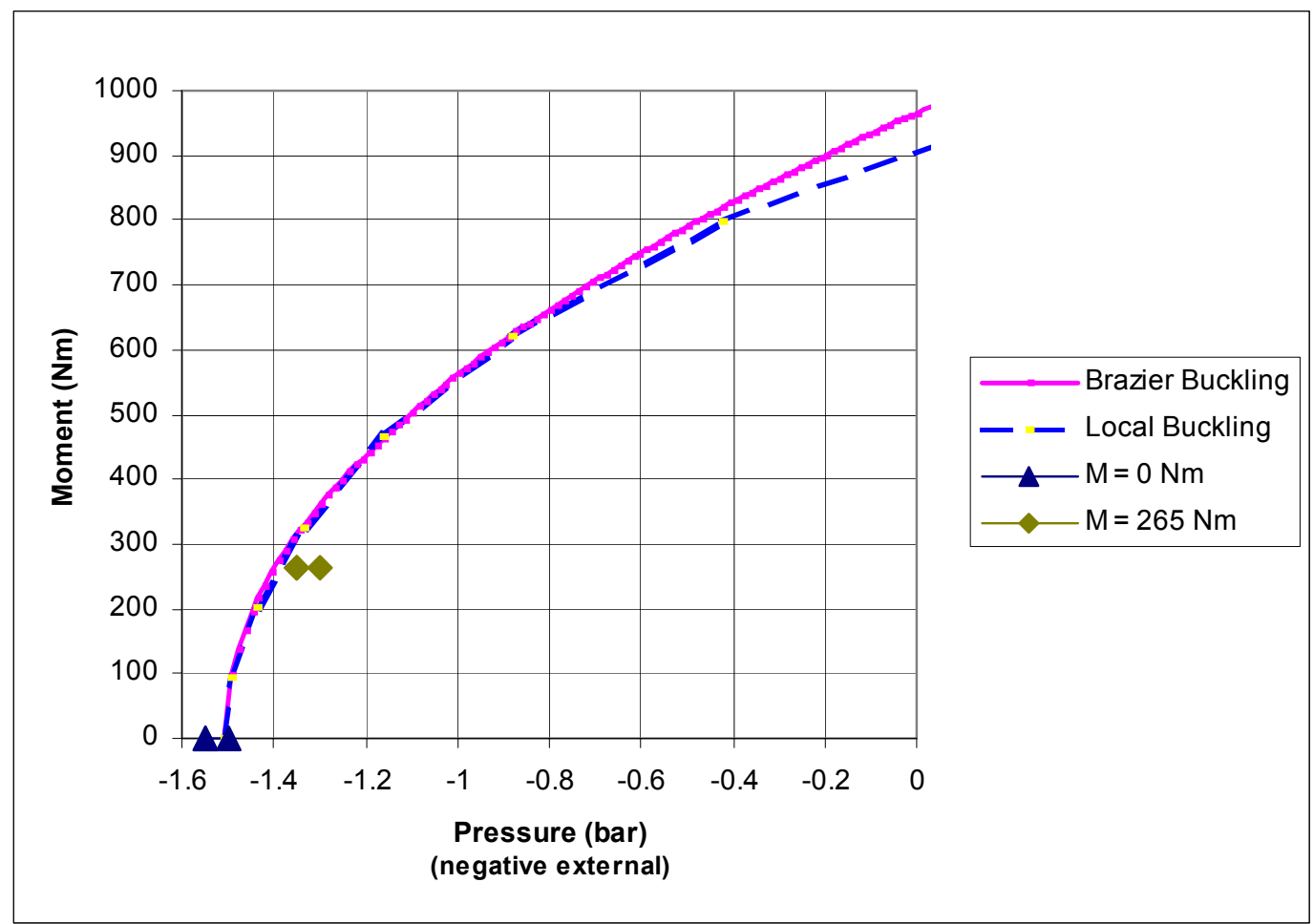

